\title{
State-dependent Dilatancy Theory and Numerical Modelling of Rockfills
}

\author{
Xiao-mei Li ${ }^{1}$, Lin Han ${ }^{2}$, Yun-fei Guan ${ }^{1}$ \\ 1. Department of Geotechnical Engineering, Nanjing Hydraulic Research Institute, Nanjing \\ 210024, P. R. China \\ 2. College of Mechanics and Materials, Hohai University, Nanjing 211100, P. R. China \\ E-mail:xmli@nhri.cn,lion_han@hhu.edu.cn,yfguan@nhri.cn
}

\begin{abstract}
The dilatancy behavior of rockfills is relate to the stress level, the initial state and particle breakage. In this paper, based on the critical state theory, the state-dependent dilatancy theory of rockfills is established, and it is introduced into the state-dependent constitutive model of coarse materials, so the state-dependent constitutive model of rockfills is formulated. According to the large-scale triaxial testing results, using the Fortran program modelling the experimental results, then, comparing the test results and simulation results, only one set parameters of state-dependent constitutive model of rockfills can reflect the strain softening and dilatancy properties of rockfills under the condition of different density, gradation and confining pressure. Therefore, the rationality of the state-dependent constitutive model of rockfills is verified.
\end{abstract}

Keywords: rockfills; dilatancy; critical state theory; constitutive model; numerical modelling.

\section{Introduction}

Dilatancy is an important engineering characteristic of granular materials. In general, volume expansion in shearing is called shear expansion, and volume shrinkage is called shear contraction [1]. Classical stress dilatancy theory (Rowe,1962) is used for describing the relationship between the dilatancy and stress ratio. In 1962, Rulong Wei [2] discussing the dilatancy theory of soil in detail, he believed that the dilatancy of granular materials is result of the particle interlocking, the main reason is particle turning over adjacent particle or having tendency in the shear process.

Base on the classical stress dilatancy theory, many scholars at home and abroad have studied the shear dilatancy behaviors of coarse materials. The dilatancy behavior of sand has been studied [3-8], and the state dependent dilatancy theory of sand has been established. Both rockfills and sand are coarse granular materials, but rockfills has many different engineering properties as large particle size, high strength, small deformation and particle breakage.

Liu $[9,10]$ studies the dilatancy behavior of rockfills by large-scale triaxial experiment, pointing out that stress path and consolidation stress are the main external factors, establishing new stress dilatancy function. Xu [11] establishes strain hardening constitutive model of rockfills, which can describe the dilatancy and contraction of rockfills. Chu [13] researches the dilatancy behavior of coarse materials, he believes that the dilatancy function of Modified Cambridge model can't reflect the dilatancy behavior of coarse materials, Row's dilatancy function underestimating its compressibility in the contraction stage, and overestimating its dilatancy behavior in the shear expansion stage, so he studies the relationship between the density, confining pressure and dilatancy by large scale triaxial experiment.

Correct understanding the dilatancy behavior is the key for establishing reasonable constitutive model. In this paper, referring to the current research results, based on the critical state theory, establishing the state dependent dilatancy theory of rockfills, which is introduced into the state-dependent constitutive model of coarse granular materials, so the state-dependent constitutive model of rockfills is formulated. A numerical simulation of largescale triaxial test is carried out by compiling Fortran program, comparing the test results and simulation results, only one set parameters of state-dependent constitutive model of rockfills can reflect the strain softening and dilatancy properties.

\section{State-dependent dilatancy theory of rockfills}

The dilatancy equation can directly reflect the relationship between the shearing deformation behavior and its influencing factors. When dilatancy ratio is greater than or equal to zero, it is shrinkage in the shearing process, 
and when dilatancy ratio is less than zero, it is dilatancy. Li [6] proposes dilatancy function of sand, which covering the void ratio and other intrinsic state variables, the dilatancy equation is as follows:

$$
d=d_{1}\left(e^{m \psi}-\frac{\eta}{M}\right)
$$

Among them,

$$
\begin{aligned}
& \psi=e-e_{\mathrm{c}} \\
& e_{\mathrm{c}}=e_{\Gamma}-\lambda\left(\frac{p^{\prime}}{p_{\mathrm{a}}}\right)^{\xi} \\
& \eta=q / p^{\prime}
\end{aligned}
$$

In which, $d$ is dilatancy ratio, $d_{1}$ and $m$ are model parameters, $\psi$ [6] is state variable, $\eta$ is stress ratio, $M$ is critical state stress ratio, $e$ is the current void ratio, $e_{\mathrm{c}}$ is the critical void ratio, $e_{\Gamma}$ is the void ratio when the effective mean normal stress is zero, $\lambda$ is the slope of critical state line, $\xi$ is material parameter, $p^{\prime}$ is effective mean normal stress, $p_{\mathrm{a}}$ is atmospheric pressure, $q$ is deviatoric stress.

Rockfills and sand are all coarse granular materials, they have similar dilatancy behaviors, classical stress dilatancy function can't reflect their deformation characteristics. But, comparing to sand, rockfills has larger particle size, easier to breakage, they have different engineering behaviors. In this paper, referring to the state dependent dilatancy theory of sand, studying the dilatancy behavior of rockfills and establishing the dilatancy function, so the state dependent dilatancy theory of coarse granular material is improved.

The dilatancy of rockfills is relate to the factors of gradation, density, stress level and particle breakage, based on the critical state theory of rockfills, the general state dependent dilatancy function of rockfills is as follows.

$$
d=f\left(D_{0}, e, \eta, C\right)
$$

Among them,

$$
\eta=q / p^{\prime}
$$

In which, $d$ is dilatancy ratio, $D_{0}$ is the initial dimension of particle distribution, $e$ is the current void ratio, $\eta$ is stress ratio, $p^{\prime}$ is effective mean normal stress, $q$ is deviatoric stress, $C$ is other internal state parameters.

In the equation (5), when the sample reach critical state, the current void ratio is critical void ratio, the current stress ratio is critical stress ratio, the shear deformation tends to be stable, and the dilatancy ratio equal to zero. In the shearing process, in the begin, it is shear contraction then is dilatancy, when the shear shrinkage is transited to the dilatancy, the dilatancy ratio is equal to zero also, but the current void ratio is not critical void ratio, the current stress ratio is phase transitional stress ratio.

According the above analysis, the state dependent dilatancy function of rockfills is similar to sand's, which is proposed by Li [6], the state dependent dilatancy function of rockfills is as follows,

$$
d=d_{0}\left(e^{m \psi}-\frac{\eta}{M}\right)
$$

Among them,

$$
\begin{aligned}
& \psi=e-e_{\mathrm{c}} \\
& e_{\mathrm{c}}=e_{\Gamma}-\lambda\left(\frac{p^{\prime}}{p_{\mathrm{a}}}\right)^{\xi} \\
& e_{\Gamma}=f\left(D, e_{0}\right)=c-a D+b e_{0} \\
& D=l+\alpha\left(\sigma_{3} / p_{\mathrm{a}}\right)+\beta D_{0} \\
& \eta=q / p^{\prime}
\end{aligned}
$$

In which, $d$ is dilatancy ratio, $d_{0}$ and $m$ are model parameters, $\psi$ [6] is state variable, $\eta$ is stress ratio, $M$ is critical state stress ratio, $e$ is the current void ratio, $e_{\mathrm{c}}$ is the critical void ratio, $e_{\Gamma}$ is the void ratio when the effective mean normal stress is zero, $\lambda_{\mathrm{c}}$ is the slope of critical state line, $\xi$ is material parameter, $p^{\prime}$ is effective mean normal stress, $p_{\mathrm{a}}$ is atmospheric pressure, $D$ is the fractal dimension, $e$ is the void ratio after consolidation, $\sigma_{3}$ is confining pressure, $D_{0}$ is the initial dimension of particle distribution, $q$ is deviatoric stress, $a, b, c, l, \alpha$ and $\beta$ are material constants.

It can be seen from equation (10) that the void ratio of sand is different from the rockfills' when the effective mean normal stress is zero, which is relate to the initial state, stress level and particle breakage. when the shearing shrinkage is transited to the dilatancy, the dilatancy ratio is equal to zero, the model parameter 
$m=\ln \left(M^{\mathrm{d}} / M\right) / \psi^{\mathrm{d}}$, in which, $M^{\mathrm{d}}$ is phase transitional stress ratio, $M$ is critical stress ratio, $\psi^{\mathrm{d}}$ is phase transitional state variable.

\section{State-dependent constitutive model of rockfills}

In order to reflect the shear deformation behavior of rockfills rationally, the newly state dependent dilatancy function of rockfills is introduced into the state dependent constitutive model of coarse granular materials, which is proposed by Li and Dafalias [17], the constitutive model of rockfills is as follows.

$$
\left\{\begin{array}{l}
\mathrm{d} q \\
\mathrm{~d} p^{\prime}
\end{array}\right\}=\left(\left[\begin{array}{cc}
3 G & 0 \\
0 & K
\end{array}\right]-\frac{h(L)}{K_{\mathrm{p}}+3 G-K \eta d} \cdot\left[\begin{array}{cc}
9 G^{2} & -3 K G \eta \\
3 K G d & -K^{2} \eta d
\end{array}\right]\right)\left\{\begin{array}{l}
\mathrm{d} \varepsilon_{\mathrm{q}} \\
\mathrm{d} \varepsilon_{\mathrm{v}}
\end{array}\right\}
$$

In which, $G$ is the elastic shear modulus, $K$ is the elastic bulk modulus, $L$ is plastic loading factor, $h(L)$ is Heaviside equation, when $L$ is greater than zero, $h(L)=1$, when $L$ is less than or equal to zero, $h(L)=0, K_{\mathrm{p}}$ is plastic modulus, other symbols are the same as the previous text.

The elastic shear modulus can be calculated by equation (14), as

$$
G=G_{0} \cdot \frac{(2.973-e)^{2}}{1+e} \cdot \sqrt{p^{\prime} \cdot p_{\mathrm{a}}}
$$

So,

$$
K=G \cdot \frac{2(1+v)}{3(1+2 v)}
$$

In which, $G_{0}$ is material constant, $e$ is the void ratio after consolidation, $v$ is Poisson's ratio.

The plastic modulus $K_{\mathrm{p}}$ can be calculated by state variable $\psi$, it can reflect the strain hardening and softening of rockfills, the expression is as follows.

$$
K_{\mathrm{p}}=h G\left(\frac{M}{\eta}-\mathrm{e}^{n \psi}\right)
$$

In which, $h G$ is hardening parameter, $h=h_{1}-h_{2} \bullet e, h_{1}, h_{2}$ and $n$ are model parameters. When the strain hardening is transited to strain softening, the plastic modulus is equal to zero, so, $n=\ln \left(M / M^{b}\right) / \psi^{b}, M^{\mathrm{b}}$ and $\psi^{\mathrm{b}}$ are the stress ratio and state variable in the strain transitional phase.

The 16 parameters of state dependent constitutive model of rockfills are calibrated by the large triaxial experimental results, which can be seen in Table 1.

Table 1 Parameters of state dependent constitutive model of rockfills

\begin{tabular}{cccc}
\hline $\begin{array}{c}\text { Elastic } \\
\text { parameters }\end{array}$ & $\begin{array}{c}\text { Particle breakage } \\
\text { parameters }\end{array}$ & $\begin{array}{c}\text { Critical state } \\
\text { parameters }\end{array}$ & $\begin{array}{c}\text { State dependent } \\
\text { parameters }\end{array}$ \\
\hline \multirow{2}{*}{$G_{0}=190$} & $l=0.744$ & $M=1.727$ & $d_{0}=2.267$ \\
$v=0.30$ & $\alpha=0.008$ & $c=0.313$ & $m=0.458$ \\
& $\beta=0.699$ & $b=0.032$ & $h_{1}=0.46$ \\
& & $b=0.527$ & $h_{2}=0.78$ \\
& & $\lambda_{\mathrm{c}}=0.013$ & $n=4.95$ \\
& $\xi=0.70$ & \\
\hline
\end{tabular}

\section{Numerical modelling}

The state dependent constitutive model of rockfills is compiled by Fortran program, using the parameters calibrated by triaxial experiments and the testing initial conditions, modeling the triaxial tests, the test results and numerical results are listed in Figure 1 4.

Comparing the results in Figure 1 4, it is demonstrated that modelling results match the testing results very well. The state dependent constitutive model of rockfills can reflect the stress and deformation characteristics under the condition of different density, gradation and confining pressure, it can also reflect the strain hardening and softening, volumetric dilatancy and shrinkage. The constitutive model of rockfills is formulated considering 
the influence of density, gradation, stress state and particle breakage, but the particle shape, composition, the particle recombination in the shearing process and the technical level of the experimenter are not considered, so the model need further exploration.

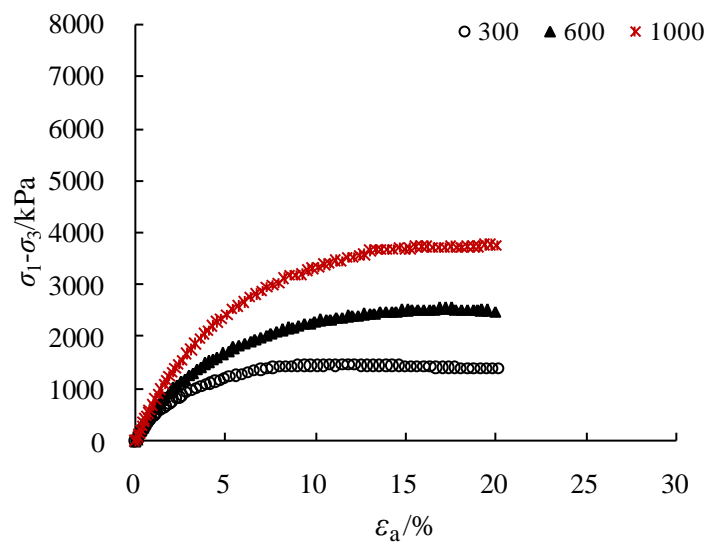

(a1) Testing results (The relative density is 0.60 )

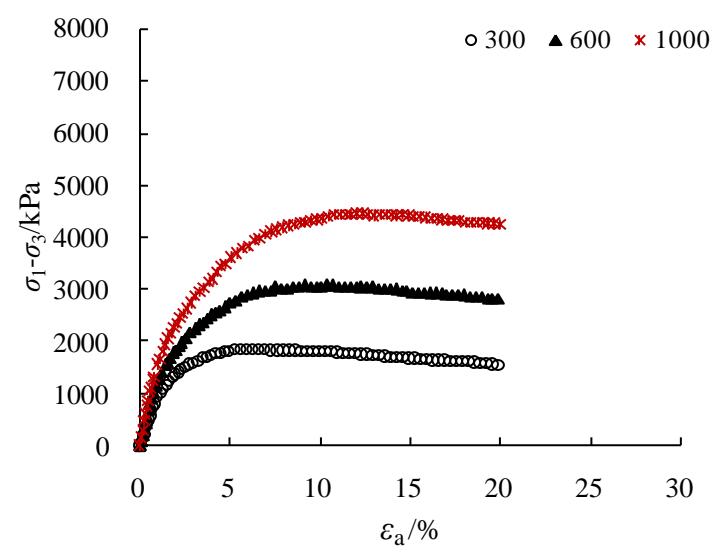

(c1) Testing results (The relative density is 0.90 )

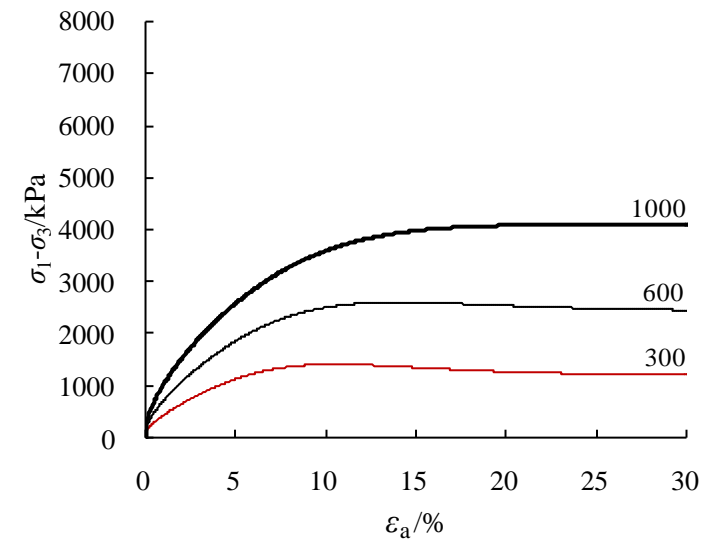

(a2) Modelling results (The relative density is 0.60 )

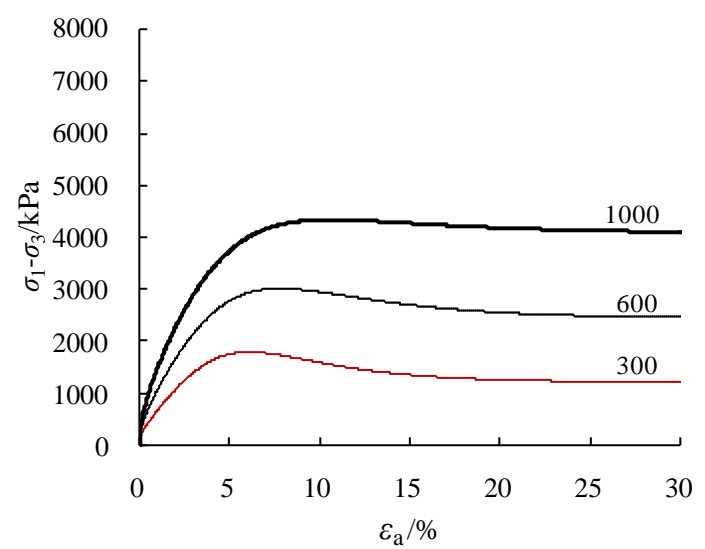

(c2) Modelling results (The relative density is 0.90 ) results and modelling results (gradation 1)

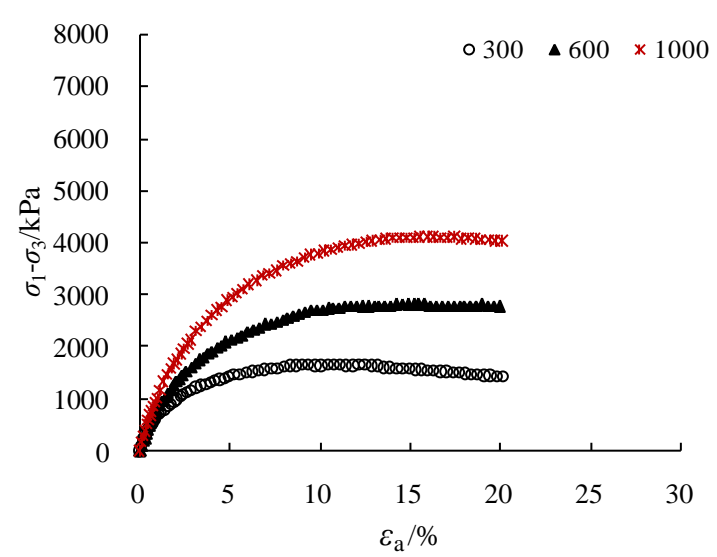

(a1) Testing results (gradation 1)

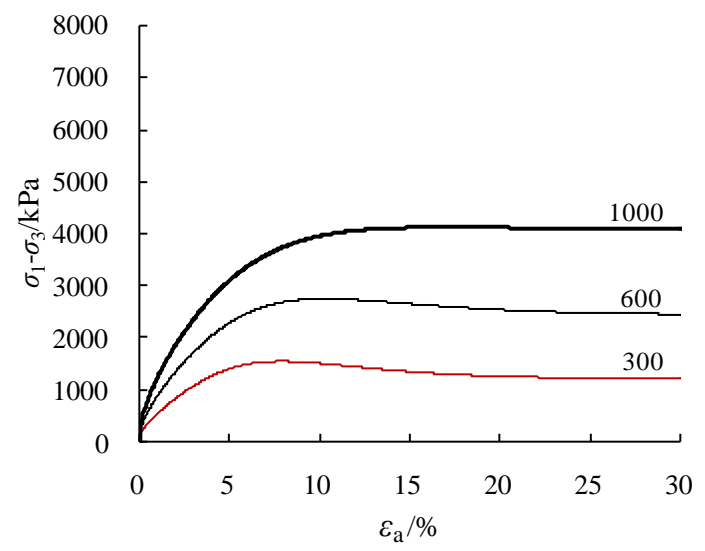

(a2) Modelling results (gradation 1) 


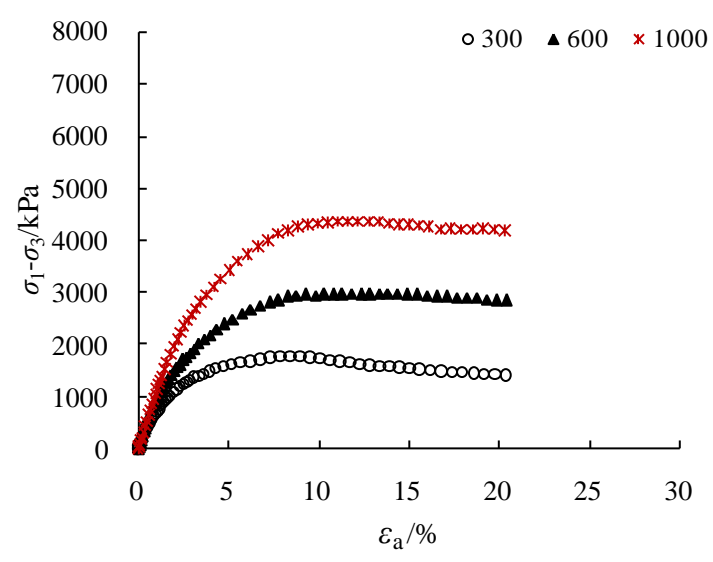

(b1) Testing results (gradation 2)

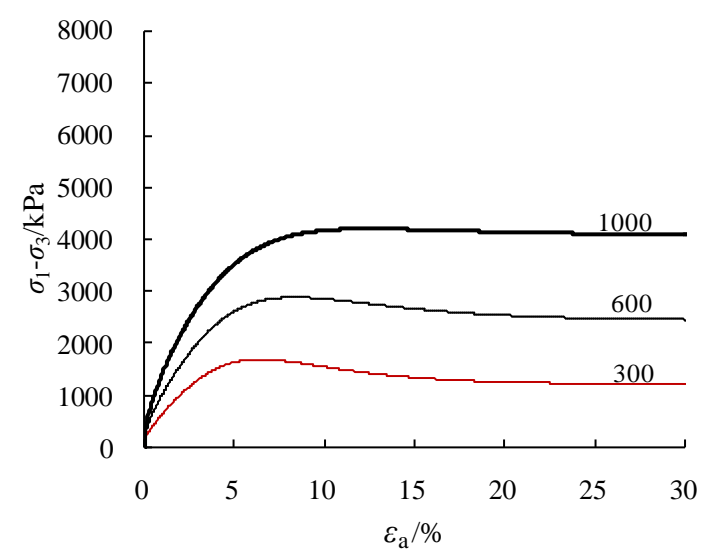

(b2) Modelling results (gradation 2)

Figure2 Deviatoric stress and axial strain of testing results and modelling results (the relative density is 0.75 )

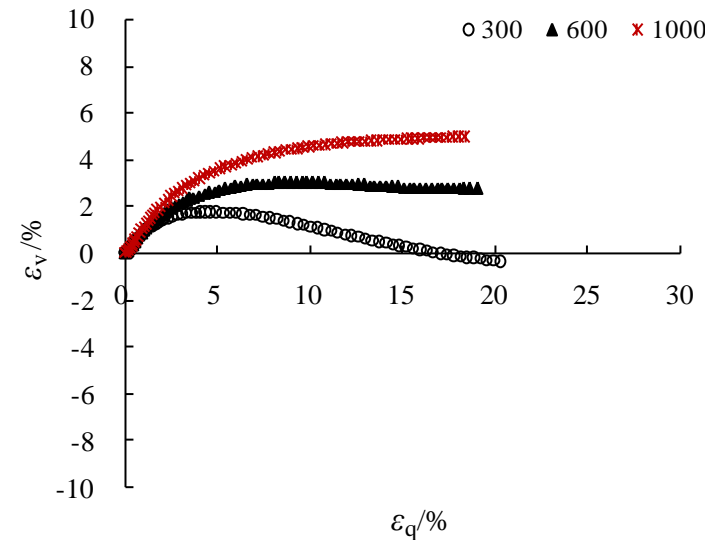

(a1) Testing results (The relative density is 0.60 )

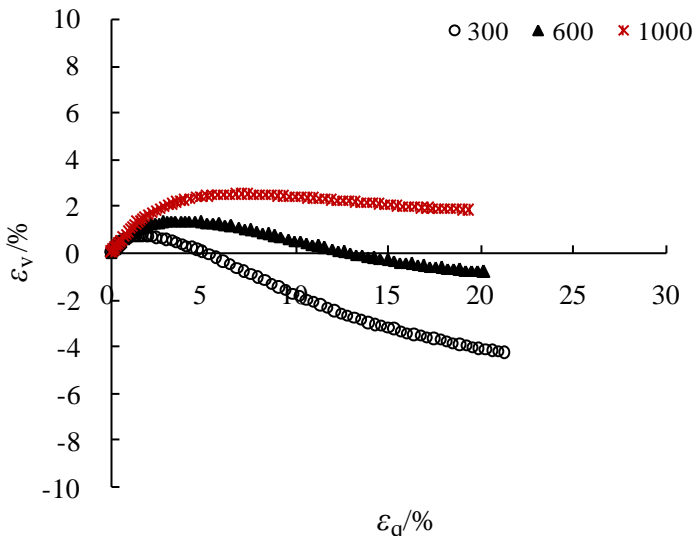

(b1) Testing results (The relative density is 0.90 )

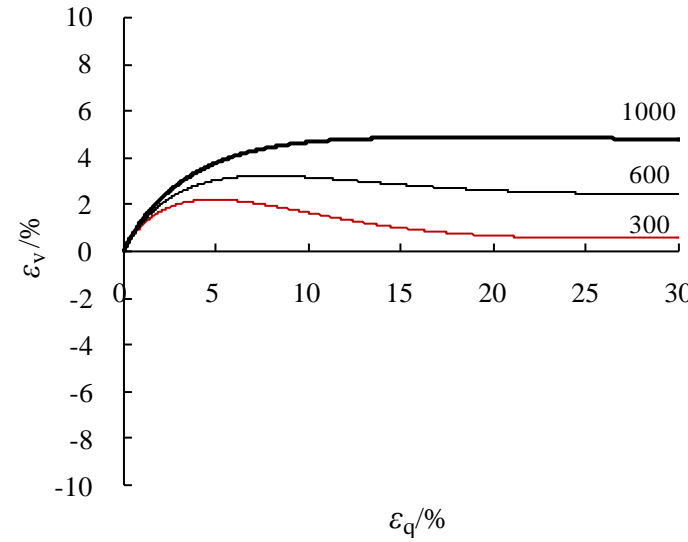

(a2) Modelling results (The relative density is 0.60 )

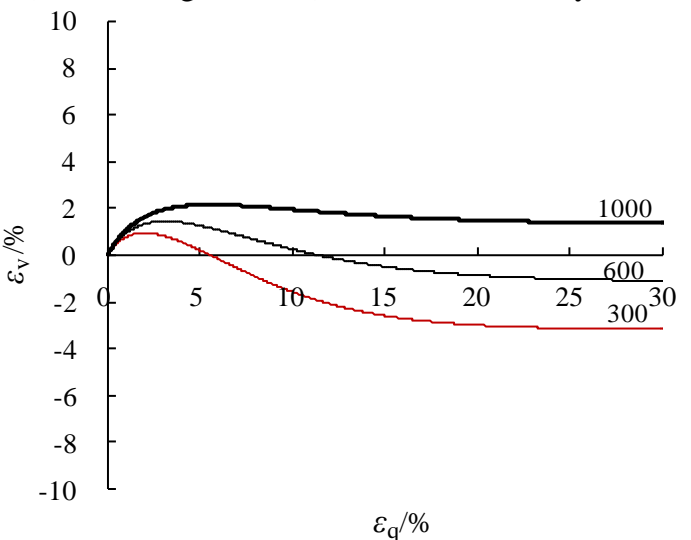

(b2) Modelling results (The relative density is 0.90 ) ing results and modelling results (gradation 1)

\section{Conclusions}

Based on the large triaxial experimental results, according the theory analysis and numerical modelling, the main conclusions are listed as follows.

(1) Based on the critical state theory of rockfills, the state dependent dilatancy theory is formulated, it is introduced into the state dependent constitutive model of coarse granular materials, so the state dependent constitutive model of rockfills is established.

(2) The large triaxial consolidation and drainage shear tests are simulated by Fortran program, comparing the testing results and numerical modelling results, it is found that the two results match very well. 
(3) The state-dependent constitutive model of rockfills using only one set parameters can reflect the stress and deformation characteristics under the condition of different density, gradation and confining pressure. It also can reflect the strain hardening and softening, volumetric dilatancy and shrinkage.

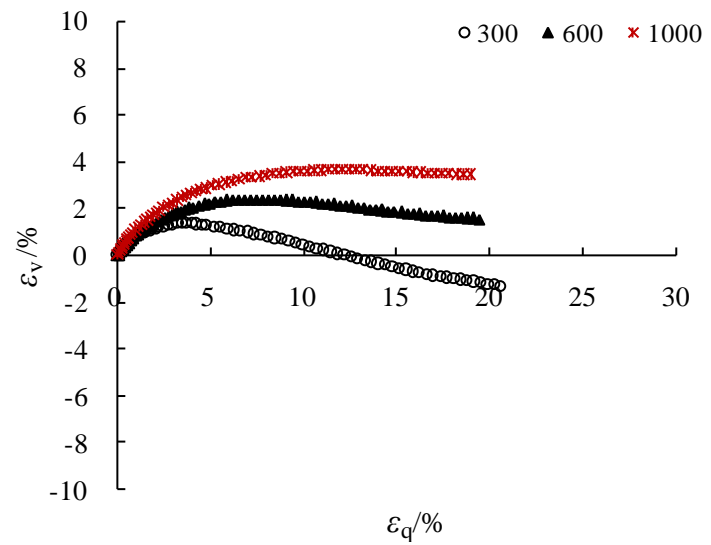

(a1) Testing results (gradation 1)

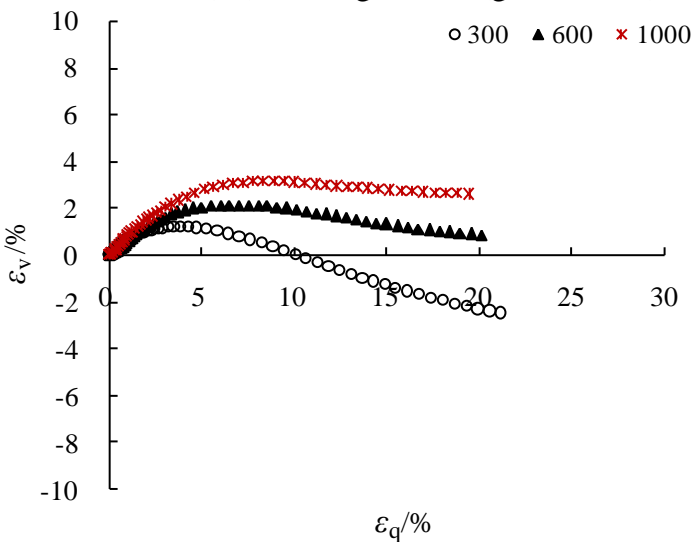

(b1) Testing results (gradation 2)

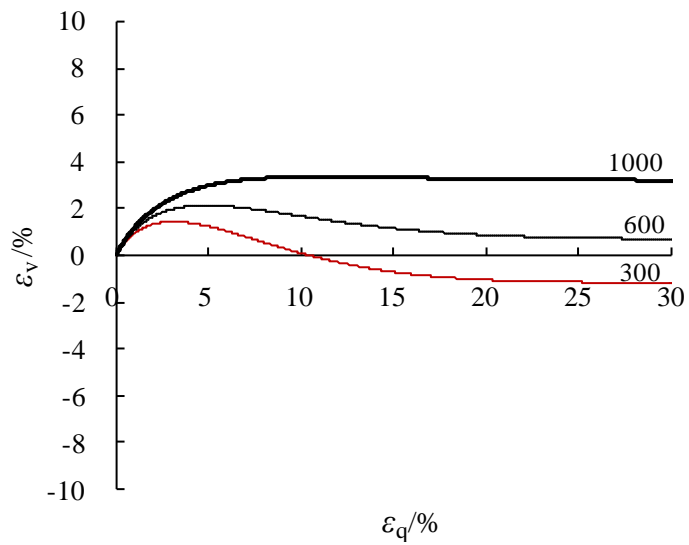

(a2) Modelling results (gradation 1)

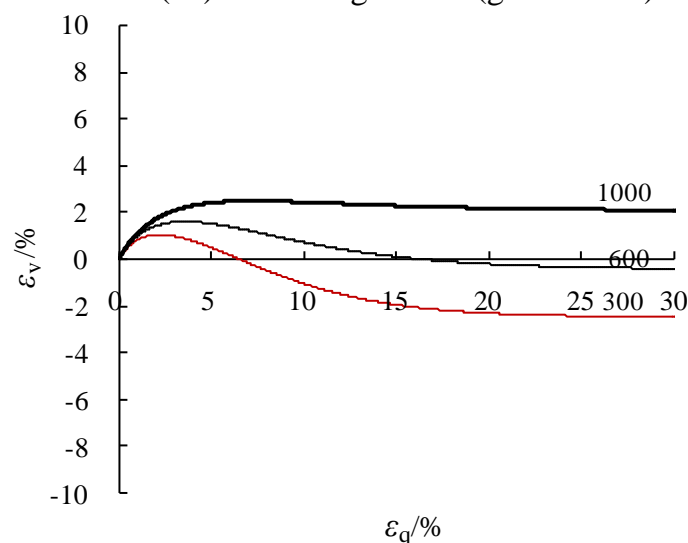

(b2) Modelling results (gradation 2)

Figure4 Volumetric strain and deviatoric strain of testing results and modelling results (the relative density is $0.75)$

\section{Acknowledgments:}

The authors would like to thank the support of the Fundamental Research Funds for the Central Public Welfare Scientific Research Institutes (Grant No. Y316022).

\section{References}

[1] Zong-ze Yin, et al. Principle on geotechnical engineering[M]. Beijing: China Water Power Press, 2007(in Chinese).

[2] Ru-long Wei. Discussion on the soil dilatancy[J]. Journal of Hydraulic Engineering, 1963(6): 31-40.

[3] K G Been, M G Jefferies. A state parameter for sands[J]. Geotechnical, 1985, 35(2): 99-112.

[4] M.T Manazari, Y.F Dafalias. A critical state two surface plasticity model for sands[J]. Geotechnical, 1997 , 47(2): 255-272.

[5] A Gajo, D M Wood. Severn-trent sand: a kinematic-hardening constitutive model: the $q-p$ formulation [J]. Geotechnique, 1999, 49: 595-614.

[6] X S Li. A sand model with state-dependent dilatancy[J]. Geptechnique, 2002, 52(3): 173-186.

[7] Zheng-yin Cai, LI Xiang-song. Deformation characteristics and critical state of sand[J]. Chinese Journal of Geotechnical Engineering, 2004, 26(5): 697- 701.

[8] Ji-zhu Sun, Xin-wen Luo. Study on a two-yield surface model with consideration of state-dependent dilatancy for calcareous sand[J]. Chinese Journal of Rock Mechanics and Engineering, 2006, 25(10): 21452149.

[9] Meng-cheng Liu, Yu-feng Gao, Han-long Liu. Study on shear behaviors of rockfill in large-scale triaxial tests under different stress paths[J]. Chinese Journal of Rock Mechanics and Engineering, 2008, 27(1): 176- 
186.

[10] Meng-cheng Liu, Yu-feng Gao, Han-long Liu. Study on shear dilatancy behaviors of rockfills in large-scale triaxial tests[J]. Chinese Journal of Geotechnical Engineering, 2008, 30(2): 205-211.

[11] Ming $\mathrm{Xu}$, Erxiang Song. Numerical simulation of the shear behavior of rockfills[J]. Computers and Geotechnics, 2009(36): 1259-1264.

[12] Jia-ming Zhang, Ming-jian Hu, Guo-qiang Hou, et al. Dilatant behavior of intensely weathered coarsegrained soils in large-scale direct shear tests[J]. Rock and Soil Mechanics, 2013, 34(S2): 67-73.

[13] Fu-yong Chu, Jun-gao Zhu, Jian-hua Yin. Study of dilatancy behaviors of coarse-grained soils in large-scale triaxial test[J]. Rock and Soil Mechanics, 2013, 34(8): 2249-2254.

[14] SL237-1999 Specification of soil test[S]. 1999(in Chinese).

[15] Jun-gao Zhu, Hou-yang Weng, Xiao-ming Wu, et al. Experimental study of compact density of scaled coarse-drained soil[J]. Rock and Soil Mechanics. 2010, 31(8): 2394-2398.

[16] Xiang-Song Li, Yannis F. Dafalias, Zhi-Liang Wang. State-dependent dilatancy in critical-state constitutive modelling of Sand[J]. Can. Geotech. J, 1999(36): 599-611.

[17] X. S. Li and Y. F. Dafalias. Dilatancy for cohesionless soils[J]. Geotechnique 50, 2000(4): 449-460.

[18] R G, Wan R G Guo. A simple constitutive model for granular soils: modified stress-dilatancy approach[J]. Computers and Geotechnics, 1998, 22(2):109-133. 